
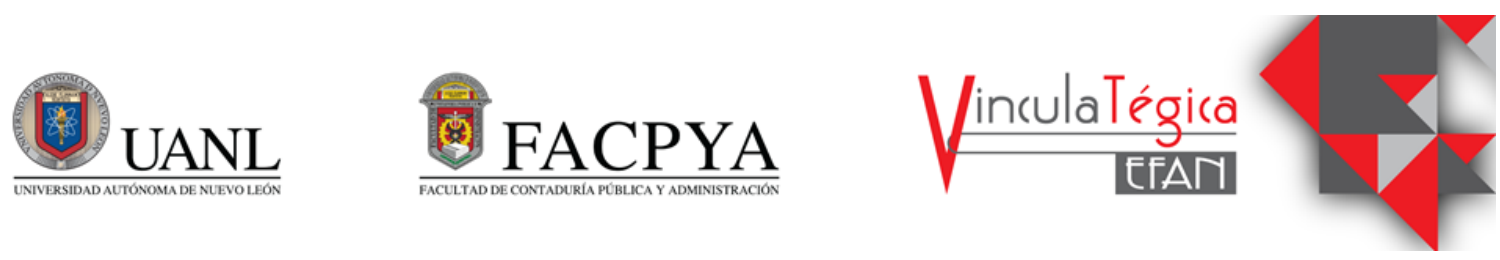

\title{
Análisis de la valoración hedónica de la vivienda del Área Metropolitana de Monterrey (2021)
}

\author{
Rubí Martínez Moreno ${ }^{1}$ y Héctor Sotelo Ahumada ${ }^{2}$ \\ ${ }^{1}$ Facultad de Arquitectura, Universidad Autónoma de N.L, arqrubimtz@gmail.com, Av.Pedro de Alba s/n, \\ Ciudad Universitaria, 66455 San Nicolás de los Garza N.L.,8183294160 \\ ${ }^{2}$ Facultad de Arquitectura, Universidad Autónoma de N.L, hector.soteloah@uanl.edu.mx, Av. Pedro de Alba \\ s/n, Ciudad Universitaria, 66455 San Nicolás de los Garza N.L.,8183294160
}

\author{
Información del artículo revisado por pares \\ Fecha de aceptación: junio-2021 \\ Fecha de publicación en línea: diciembre-2021 \\ DOI: https://doi.org/10.29105/vtga7.1-139
}

\begin{abstract}
Resumen
El objetivo de esta investigación es analizar el mecanismo de valoración hedónica de las áreas verdes en la vivienda en el área metropolitana de Monterrey (AMM). La hipótesis sostiene que, si se vincula un valor económico a las áreas verdes, se generaran a su vez, incentivos para impactar positivamente al entorno natural y social de la zona metropolitana de Monterrey. Se utilizan los resultados de la aplicación de una encuesta semiestructurada de corte transversal para el primer trimestre de 2021, se efectúa un análisis descriptivo y de frecuencia, con la finalidad de reflejar la importancia que la sociedad brinda a las áreas verdes dentro de sus propiedades. El principal resultado muestra que la población que no tiene áreas verdes accedería a crear un espacio ajardinado o arborizar dentro de su predio si la estimación porcentual de su valor del área verde es igual o superior al $10 \%$. La primera sección del trabajo presenta la introducción, la segunda revisa el marco teórico; en el tercer apartado se analiza la metodología; en seguida se presentan los resultados y finalmente, se concluye.
\end{abstract}

Palabras clave: precios hedónicos, áreas verdes, Área Metropolitana Monterrey.

\footnotetext{
Abstract

The objective of this research is to analyze the hedonic valuation mechanism of green areas in housing in the metropolitan area of Monterrey
}

(AMM). The hypothesis maintains that, if an economic value is linked to green areas, incentives will be generated to impact positively the natural and social environment of the Monterrey metropolitan area. The results of the application of a cross-sectional semi-structured survey for the quarter of 2021 were used, a descriptive and frequency analysis is carried out, in order to reflect the importance that society gives to green areas within its properties. The main result shows that the population that does not have green areas would agree to create a landscaped space or trees within their property if the percentage estimate of its value is equal to greater than $10 \%$. The first section of the work presents the introduction, the second reviews the theoretical framework; the third section analyzes the methodology; then the results are presented and finally, it is concluded.

Keywords: hedonic prices, green areas, Metropolitan Area Monterrey.

JEL: G1, G10, G12.

\section{INTRODUCCIÓN}

La correcta planeación de las ciudades conlleva a comunidades que promueven el crecimiento y desarrollo económico, lo que, a su vez, otorga bienestar a sus ciudadanos. La Organización de las Naciones Unidas, ONU, (2019) señala que las medidas encaminadas a lograr los objetivos climáticos todavía no 
avanzan a la velocidad ni en la escala necesarias para hacer frente a afrontar la emergencia climática.

Según con el Acuerdo de París adoptado por la conferencia de las Naciones Unidas sobre el cambio climático (ONU, 2015), es necesario que las emisiones de gases de efecto invernadero desciendan $7.6 \%$ cada año a partir de 2020. La División de Estadística de las Naciones Unidas, UNSD (2021) establece que los gobiernos y las empresas deben utilizar las lecciones aprendidas y las oportunidades que surgen de esta crisis ambiental para acelerar la transición hacia economías y sociedades con bajas emisiones de gases de efecto invernadero, sustentables y resilientes.

El objetivo central de la investigación es analizar la importancia del cumplimiento de las normativas en base a los lineamientos de construcción municipales y de infraestructura al momento de valuar una propiedad desde la perspectiva de una dependencia encargada de valuar propiedades. Es por ello por lo que se sostiene que, al generar un valor económico a las áreas verdes, se derivan a su vez, incentivos para impactar positivamente al entorno natural y social. Lo anterior, emerge como una oportunidad para mitigar los efectos de la crisis ambiental en la que Nuevo León, México y el mundo se hallan inmersos. Es interés de esta investigación responder al cuestionamiento de que, si se diera una ponderación significativa a la inserción de áreas verdes en nuevas construcciones como ampliaciones en las existentes durante la evaluación de estas, ¿mejoraría las condiciones climáticas del entorno?

En el estado de Nuevo León cada municipio conserva sus lineamientos en los cuales se establece que, dependiendo del tipo de construcción, metros cuadrados del predio, desplante del edificio, entre otros factores, se debe cumplir con un porcentaje de Coeficiente de Área Verde (CAV) para poder documentar un proyecto y así tramitar un permiso de construcción o trámites varios, "Reglamentos de zonificación y uso de suelo" (2019), sin embargo, el conocimiento sobre estos lineamientos hacia los habitantes, es escaso.

\section{MARCO TEÓRICO}

Uno de los aspectos más importantes a tratar a lo largo de la investigación es conocer los factores que influyen en la determinación de una valuación de una propiedad desde la perspectiva de la valoración hedónica y los efectos sobre de estos aspectos ambientales la vivienda y su entorno. Asimismo, intentar explicar a las personas tomar más a consideración estos factores ambientales al momento de elegir una propiedad.

El concepto de precios hedónicos parte de la idea que el conjunto de características que componen un bien heterogéneo tienen un reflejo en su precio de mercado. Por ello, se asume que el precio de dicho bien puede ser descompuesto en función de sus diferentes atributos y, por tanto, se puede asignar un precio implícito a cada uno de dichos atributos, ha sido muy usado para estudiar el efecto de la contaminación del aire en los precios de viviendas (Asatch, 2015). Cuando se venden en el mercado bienes diferenciados por el atributo de calidad esta variación puede usarse en modelos hedónicos si existe un adecuado monitoreo de la variación de la calidad del aire.

La teoría de precios hedónicos asigna valor a un bien raíz, entendido como un conjunto de atributos (superficie, aptitud de uso del suelo, calidad de la construcción, diseño interior y exterior, áreas verdes, ubicación, características del vecindario, en el caso de viviendas o construcciones de unidades habitacionales) y, en función de cada uno de ellos, se determina sus valoraciones individuales y, por ende, demandas implícitas (Moreno y Alvarado, 2011).

Este método de precios hedónicos permite valorar intangibles y bienes $\mathrm{o}$ servicios no mercadeables como el medio ambiente para establecer la relación funcional entre el precio del bien raíz y sus respectivas características (Lever, 2000). Esta metodología es común en el análisis de bienes raíces, mercados laborales, mercado de autos, entre otros (Mendieta, 2001).

La conducta económica consiste en una serie de elecciones entre las distintas alternativas posibles, lo que implica un juicio, una valoración. El criterio para determinar qué 
alternativa se prefiere supone que el sujeto económico elegirá aquella que le proporcione más satisfacción o, lo que es lo mismo, buscará la situación óptima.

La relación entre crecimiento y medio ambiente ha cobrado gran fuerza para los estudiosos de la economía en los últimos años, como es el caso del estudio "Apertura comercial y calidad ambiental: evidencia internacional" realizado en el año 2016 a 98 países debido, fundamentalmente, a la creciente degradación de los recursos naturales que acompaña al incremento de la producción a escala mundial (Figueroa, 2013).

$\mathrm{Si}$ bien algunos recursos naturales poseen un precio en el mercado, este no contempla, en la mayoría de los casos, las virtudes y funciones de dicho recurso, lo que evidentemente, añade mayor valor económico. En este contexto, esta omisión afecta el diseño e implementación de políticas públicas asociadas al manejo del recurso natural en cuestión. El valor de los recursos naturales y el medio ambiente radica en que cumplen al menos con cuatro funciones, percibidas positivamente por la sociedad (Rangel et al., 2013). Ellas son:

1. Forman parte de la función de producción de gran cantidad de bienes y servicios económicos;

2. Cumplen funciones ambientales, como protección del suelo y de los recursos de agua, cuyos servicios son demandados por la sociedad;

3. Actúan como receptores de residuos y desechos de diversos tipos;

4. Constituyen un sistema integrado que proporciona los medios elementales para sostener toda clase de vida.

Cerda, (2003) plantea que es importante destacar que no se está valorando el ambiente ni la vida, sino que se valoran las preferencias de las personas ante cambios en las condiciones del ambiente y con respecto a cambios en los riesgos que enfrentan.

Una parte de la profesión (englobada bajo el epíteto de economía ambiental) entiende que el medio ambiente puede regirse por los mismos criterios que el resto de los recursos, insistiendo en la perspectiva del mercado y, por ende, en el de los derechos de propiedad y los precios. Cerda, (2003)

Por otro lado, los defensores de la economía ecológica como K.E. Bulding, 1966, optan por un replanteamiento global de la disciplina, una especie de vuelta a los orígenes, retomando los planteamientos de los fisiócratas para enfocar la economía desde una perspectiva ecosistémica, en la que la propiedad privada tiene un menor papel protagonista y se prima la visión ecológica (Aguilera, 1992).

\section{MÉTODO}

Para determinar cómo influyen los aspectos ambientales en la valuación de las viviendas en la zona metropolitana de Monterrey se utilizaron resultados que emanaron de la aplicación de un instrumento denominado "Encuesta para residencias del área metropolitana de Monterrey, 2021", aplicada a los municipios: General Escobedo, San Nicolás, Monterrey, Guadalupe, Apodaca, García, Juárez, San Pedro Garza García y Santa Catarina de manera virtual. El muestreo utilizado fue realizado en un periodo de estudio del primer trimestre en el año 2021 utilizando un muestreo no probabilístico por conveniencia. Esto con el fin de recolectar evidencias claras con base al uso y los objetivos de las teorías generales y sustantivas que fundamentarán y definirán el objetivo teórico de esta investigación.

Por otro lado, el objetivo central de la investigación es analizar la importancia del cumplimiento de las normativas al momento de valuar una propiedad. Es por ello por lo que se sostiene que, al generar un valor económico a las áreas verdes, se derivan a su vez, ventajas para impactar positivamente al entorno natural y social.

Se partió de identificar la unidad de análisis para esta investigación, es decir, los residentes del AMM. Se aplicó una encuesta en la que se consultó esencialmente: aspectos de áreas verdes e infraestructura de las zonas con el objeto de detectar la manera en que la población del AMM asigna valor a las áreas verdes en las unidades habitacionales nuevas y ocupadas. Los resultados de la encuesta aplicada se presentan mediante un análisis de 
frecuencia y tablas cruzadas con el objetivo de cuantificar los efectos de las variables explicativas sobre el valor hedónico de las viviendas respecto de los aspectos ambientales.

\section{RESULTADOS}

A continuación, se presentan las distintas situaciones, opiniones y servicios que las personas con residencia dentro del AMM cuentan en su vivienda o en el entorno en el que se encuentran situados.

En la siguiente tabla se pueden apreciar los datos generales de las 240 personas entrevistadas con residencia dentro del AMM en la cual se puede observar que la cantidad más pronunciada de personas que respondieron la encuesta, son mujeres y mayoritariamente a personas solteras. Se entrevistaron a 173 jóvenes de entre 16 a 26 años donde se concentra el $72.08 \%$ de la población consultada, con un nivel de escolaridad concluida de licenciatura $(60 \%)$ un salario promedio de entre $\$ 5,001$ - $\$ 15,000$. Dentro de las encuestas es importante mencionar que el municipio en el que más se observó respuesta fue en General Escobedo $(24.16 \%)$ y área con menor cantidad de respuestas es Juárez $(0.83 \%)$.

Tabla 1. Información general de residentes del AMM, 2021.

\begin{tabular}{|c|c|c|c|}
\hline$\overline{\text { Variable }}$ & Categoría & Cantidad & Porcentaje \\
\hline Género & $\begin{array}{l}\text { Hombre } \\
\text { Mujer } \\
\text { Total: } 240\end{array}$ & $\begin{array}{l}108 \\
132\end{array}$ & $\begin{array}{l}45 \% \\
55 \%\end{array}$ \\
\hline$\overline{\text { Estado Civil }}$ & $\begin{array}{l}\text { Solteros } \\
\text { Casados } \\
\text { Divorciados } \\
\text { Total: } 240\end{array}$ & $\begin{array}{l}195 \\
43 \\
2\end{array}$ & $\begin{array}{l}81.25 \% \\
17.91 \% \\
0.83 \%\end{array}$ \\
\hline$\overline{\text { Edad }}$ & $\begin{array}{l}16 \text { a } 26 \text { años } \\
27 \text { a } 36 \text { años } \\
37 \text { a } 46 \text { años } \\
47 \text { a } 58 \text { años } \\
\text { Total: } 240\end{array}$ & $\begin{array}{l}173 \\
46 \\
13 \\
8\end{array}$ & $\begin{array}{l}72.08 \% \\
19.16 \% \\
5.41 \% \\
5.71 \%\end{array}$ \\
\hline Escolaridad & $\begin{array}{l}\text { Secundaria } \\
\text { Preparatoria } \\
\text { Licenciatura } \\
\text { Maestría } \\
\text { Total: } 240\end{array}$ & $\begin{array}{l}4 \\
25 \\
178 \\
33\end{array}$ & $\begin{array}{l}1.66 \% \\
10.40 \% \\
74.16 \\
13.75\end{array}$ \\
\hline Municipio de residencia & $\begin{array}{l}\text { San Nicolás } \\
\text { Gral. Escobedo } \\
\text { Apodaca } \\
\text { García } \\
\text { Guadalupe } \\
\text { Monterrey } \\
\text { Santa Catarina } \\
\text { San Pedro } \\
\text { Juárez } \\
\text { Total: } 240\end{array}$ & $\begin{array}{l}32 \\
58 \\
48 \\
6 \\
29 \\
50 \\
10 \\
5 \\
2\end{array}$ & $\begin{array}{l}13.33 \% \\
24.16 \% \\
20 \% \\
2.5 \% \\
12.08 \% \\
20.83 \% \\
4.16 \% \\
2.083 \% \\
0.83 \%\end{array}$ \\
\hline
\end{tabular}




\begin{tabular}{llll}
\hline Ingreso Mensual: & Menos de $\$ 5,000 \mathrm{MXN}$ & 36 & $15 \%$ \\
& $\$ 5,001-\$ 15,000 \mathrm{MXN}$ & 112 & $46.66 \%$ \\
& $\$ 15,001-\$ 25,000 \mathrm{MXN}$ & 74 & $30.83 \%$ \\
& Mayor de $\$ 30,000 \mathrm{MXN}$ & 15 & $6.25 \%$ \\
& & \\
\hline
\end{tabular}

Fuente: Elaboración propia con base en la Encuesta para residencias del área metropolitana de Monterrey, 2021

El $37 \%$ de las personas entrevistadas consideran que no cuentan con una buena calidad visual y $68 \%$ respecto a contaminación auditiva. También se detectó que la calidad de pavimentación en algunas colonias es deficiente $(57 \%)$

Gráfica 1. Aspectos contaminantes en la zona de residencia del AMM, 2021.

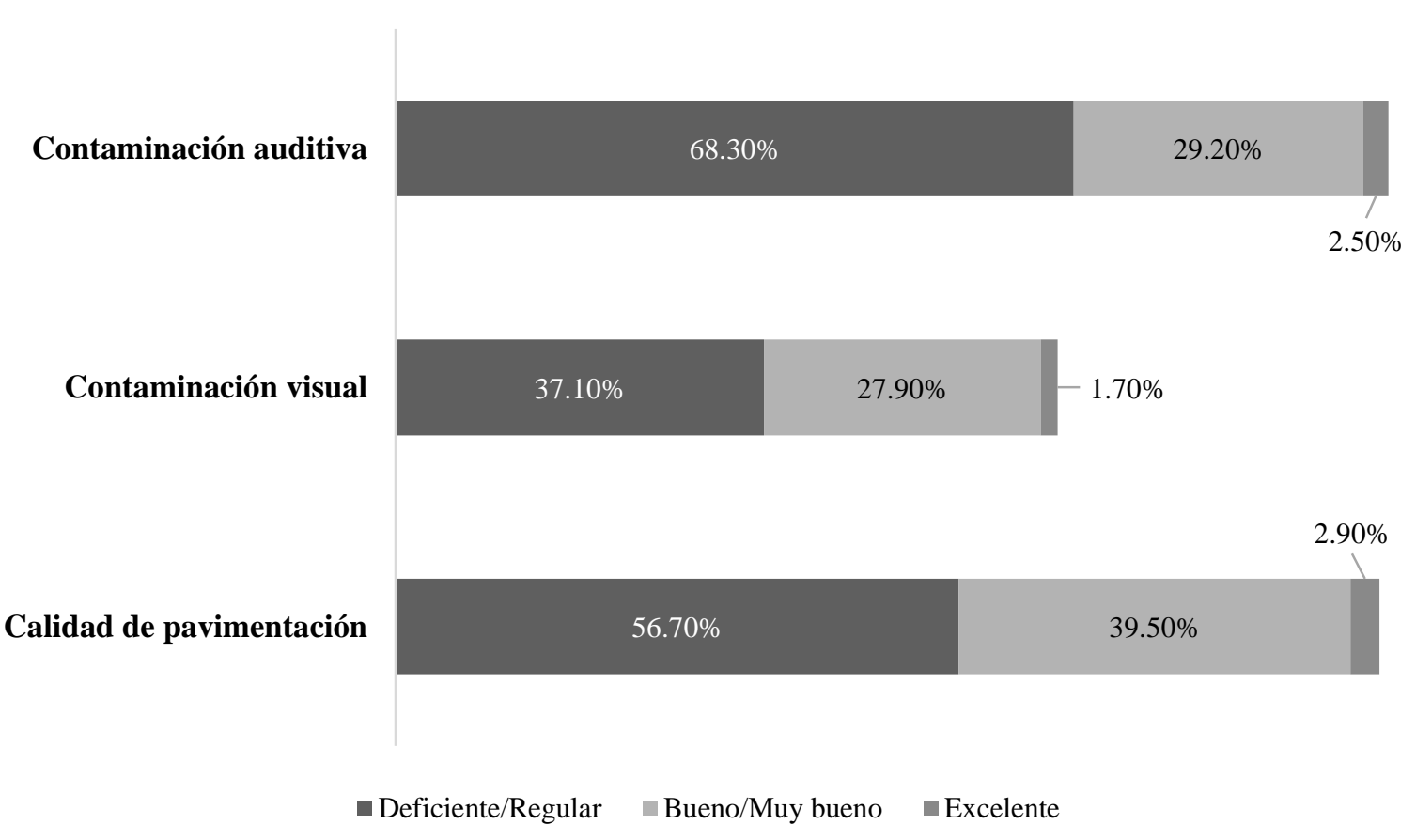

Fuente: Elaboración propia con base en la Encuesta para residencias del área metropolitana de Monterrey, 2021.

De acuerdo con los datos recabados, el $59 \%$ de las personas entrevistadas cuenta con área verde asignada en su propiedad, en su mayoría con más de $5 \mathrm{~m}^{2}(56 \%)$. Además, es posible observar que la mayor parte de estas personas declaró que estaría dispuesta a aumentar esta área si estuviera dentro de sus posibilidades $(37.90 \%)$ y de las personas que aún no cuentan con espacios verdes asignados, el $77.10 \%$ aceptaría adecuarlo de ser posible.

En promedio el $96.3 \%$ de las personas entrevistadas consideran que, si todos contribuimos con un área verde asignada en nuestra propiedad, la calidad de aire mejoraría notablemente. 
Gráfica 3. Influencia de las áreas verdes en el AMM, 2021.

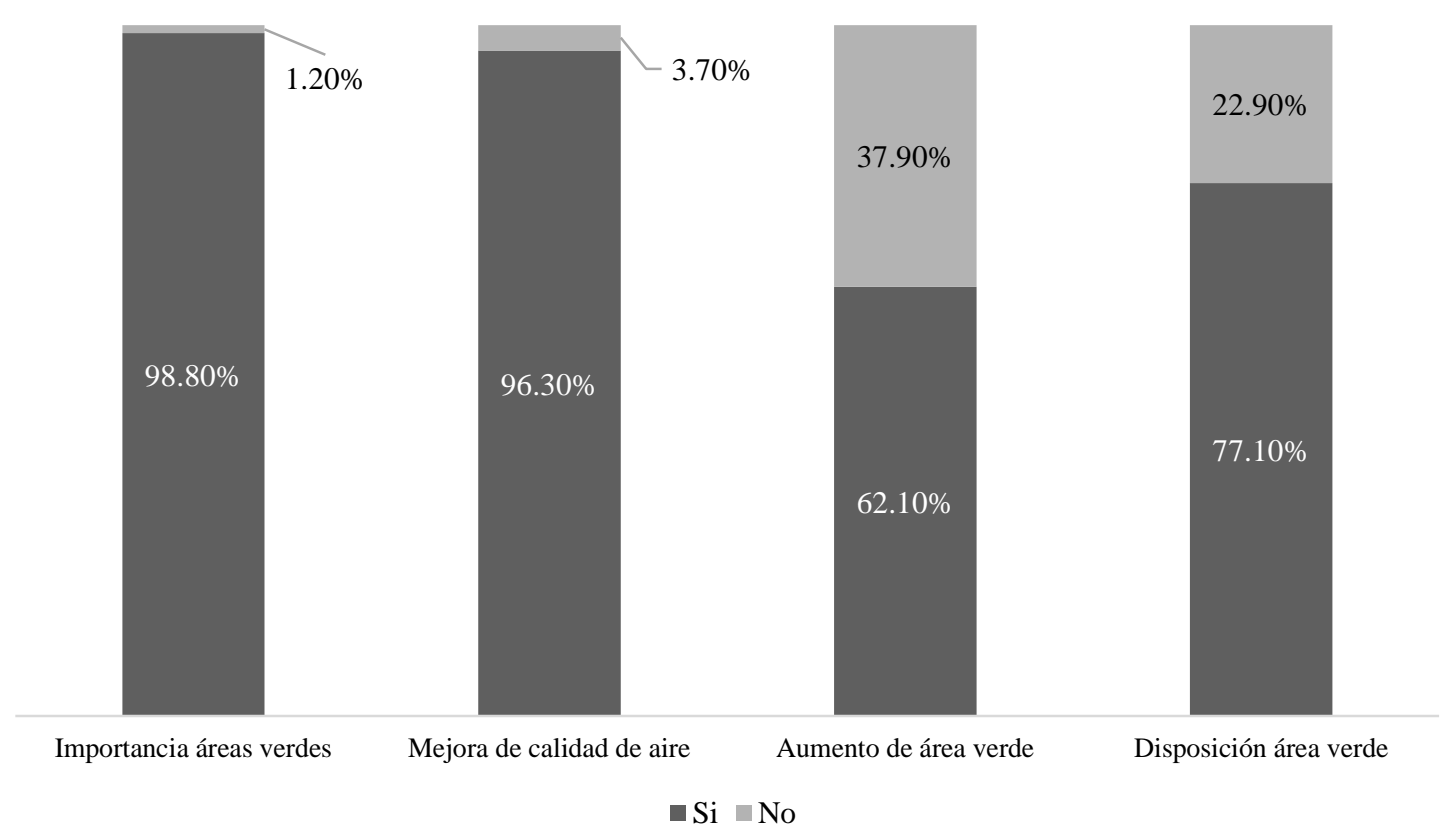

Fuente: Elaboración propia con base en la Encuesta para residencias del área metropolitana de Monterrey, 2021

Respecto a las variables de seguridad, infraestructura y servicios urbanos, se encuentra que muy pocas personas residentes dentro del AMM que cuentan con servicio de recolección de aguas residuales $(42.90 \%)$. Con respecto al nivel del índice de seguridad en las colonias, el $31.30 \%$ de la población considera que no se encuentran viviendo en una zona segura y un $38.8 \%$ no cuentan con suficientes rutas de transporte colectivo. En cuanto a instituciones educativas y de salud aún hay algunas zonas rezagadas en las que estos servicios no se encuentran cercanos $(8.80 \%$ y $17.90 \%$ respectivamente). 
Gráfica 4. Servicios urbanos en zonas de residencia del AMM, 2021.

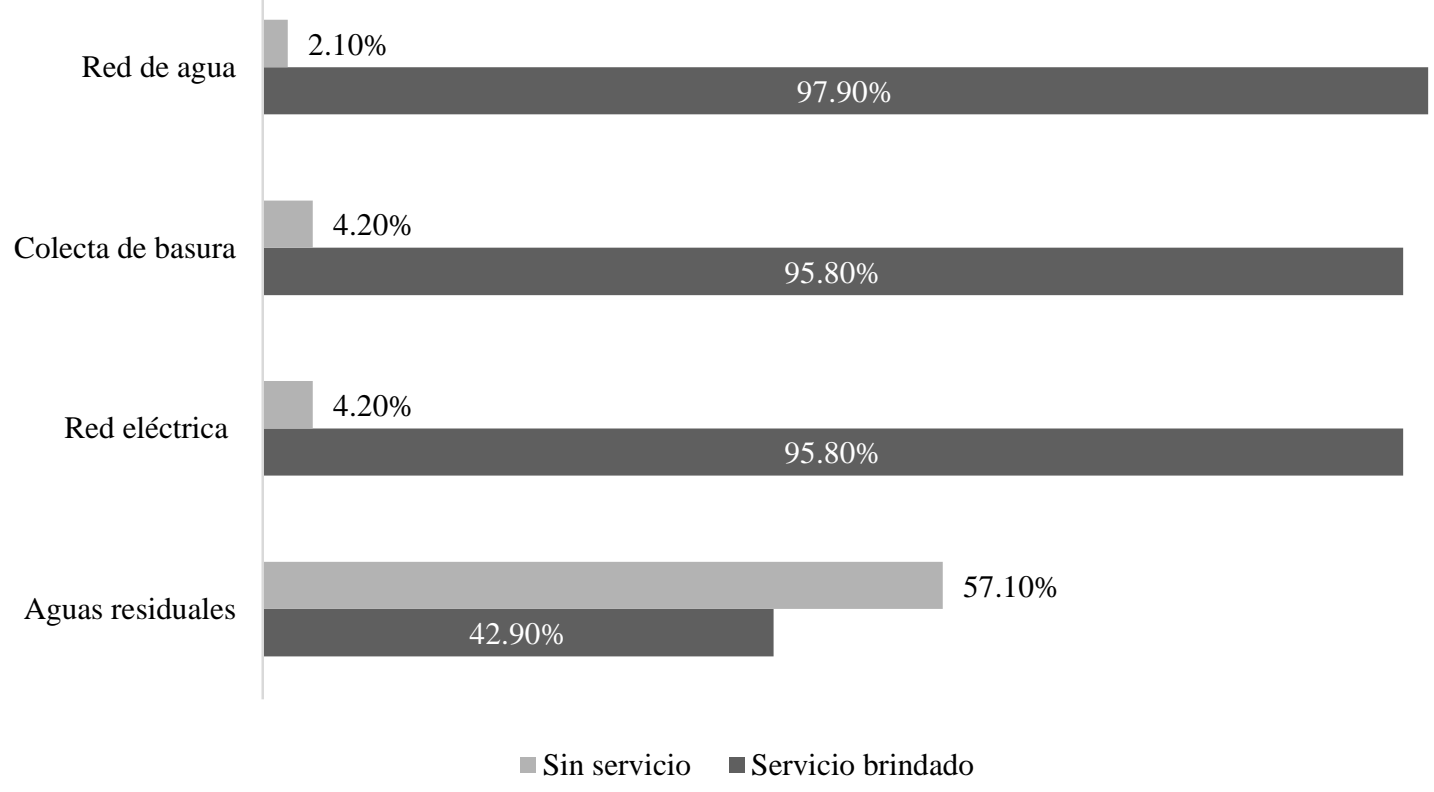

Fuente: Elaboración propia con base en la Encuesta para residencias del área metropolitana de Monterrey, 2021.

Gráfica 5. Infraestructura y seguridad

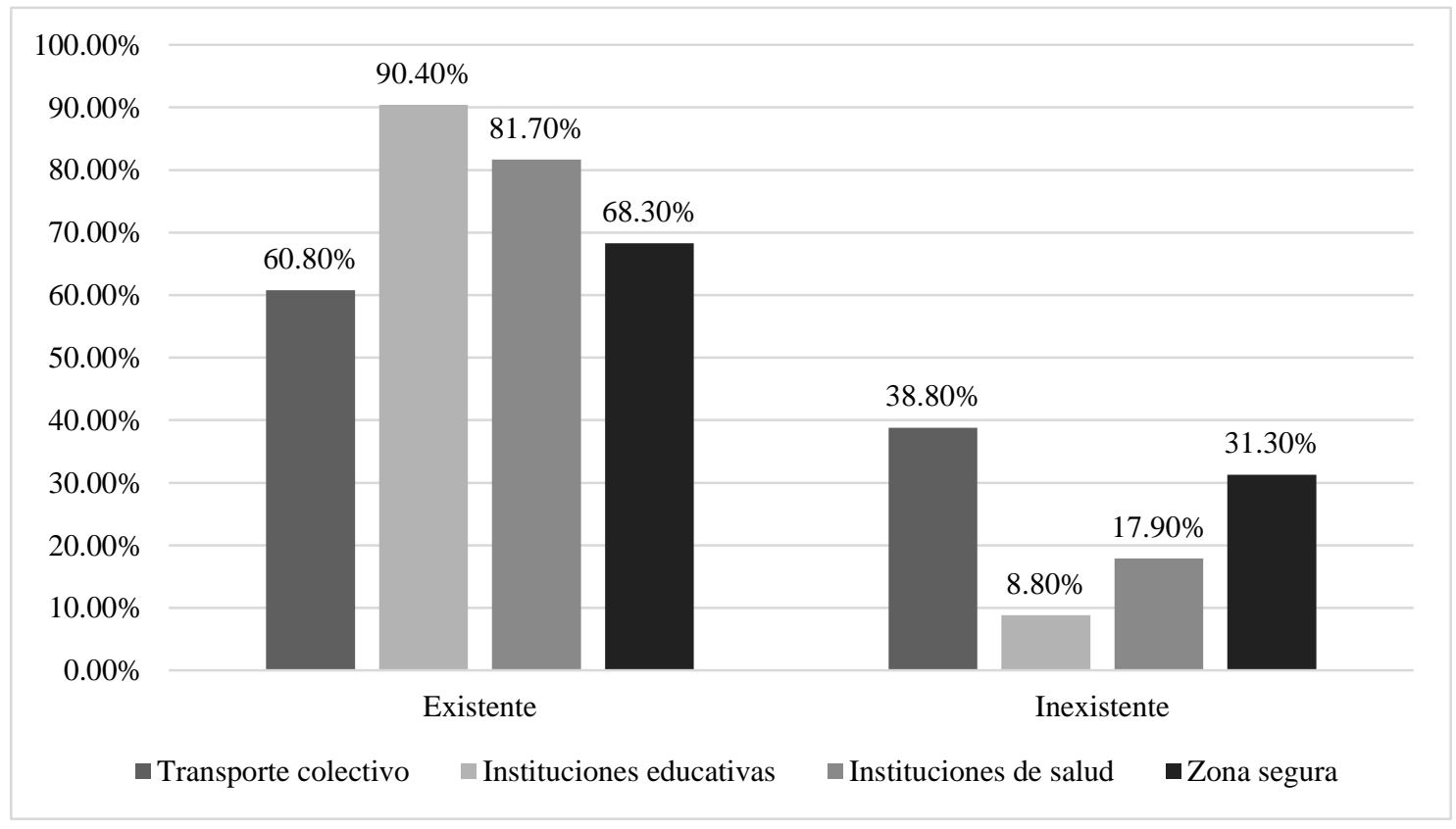

Fuente: Elaboración propia con base en la Encuesta para residencias del área metropolitana de Monterrey, 2021. 


\section{CONCLUSIONES}

Con base a los resultados obtenidos a través de la aplicación de la encuesta semiestructurada de corte transversal en el AMM, se verificó la importancia que la sociedad asocia a ciertos aspectos con doble propósito: $i$ ) reducción del impacto ambiental y ii) aumento de su valor patrimonial. El principal resultado a tratar es la accesibilidad que tienen los encuestados, puesto que el $77.10 \%$ de los ellos considerarían ampliar o adecuar un espacio verde en su vivienda. Además, destaca que las personas asumen que el impacto sería positivo en términos ambientales si todos contribuyéramos con los metros cuadrados correspondientes de área verde por vivienda recomendados. Eso a su vez, nos hace ver la importancia que la sociedad le brindaría a las áreas verdes si se les expone la problemática en la cual nos encontramos y la manera en la cual se podría aportar como habitantes.

Por otro lado, la infraestructura básica es de suma importancia para los aspectos ambientales y el aumento de valor de las viviendas, ya que, sin los servicios necesarios y adecuados, podríamos afectar no solo el medio ambiente, sino que también la valoración del inmueble y la calidad de vida de las personas.

En cuanto a infraestructura se refiere, sobresale el caso del servicio de aguas residuales, puesto que este mecanismo se usa para el riego de los parques y jardines públicos en algunos municipios, como son San Pedro Garza García y Monterrey. Sin embargo, los resultados arrojaron que el $57.10 \%$ de las personas que residen dentro del AMM no cuenta con este servicio.

De las personas entrevistadas residentes en los municipios considerados en la muestra, uno que brinda el riego con aguas residuales es San Pedro Garza García, el cual según el reporte del estado de la calidad de aire en el AMM (Secretaría de desarrollo sustentable N.L, 2021), en este municipio se encuentra un índice de calidad de aire (ICA) de moderado a bueno, entre los 59 ICA y los 38 ICA, debido a que las viviendas de este municipio prioriza el cuidado de áreas verdes públicas y privadas, garantizando el cumplimiento de metros cuadrados en cada una de sus obras civiles y, por su puesto, en las viviendas.

Derivado de este resultado se tiene claro que existe un servicio en el cual se podría atacar de manera directa y el beneficio ambiental sería aún mayor.

Por otro lado, y fuera del ámbito ecológico, podemos ver que la mayoría de las zonas del AMM cuenta con los servicios necesarios en cuanto a instituciones de salud, educativas, transporte público y seguridad. Sin embargo, los datos recabados aún marcan una pequeña negativa en los aspectos ambientales y de servicios que se puede tomar como área de oportunidad para una mejora continua.

Si le diéramos más importancia a la calidad de la infraestructura, servicios, cumplimiento en los lineamientos municipales e incluso tomando en cuenta la seguridad y las instituciones de servicios básicos, la estrategia de política pública de mejorar el entorno para contribuir al impacto medio ambiental, la valorización de las propiedades podrían incrementarse, es decir, se habrían creado y aprovechado los incentivos que la estrategia de asignación de valor a las áreas verdes brinda a áreas metropolitanas como las de Monterrey, Nuevo León. 


\section{REFERENCIAS}

Asatch, (2 septiembre 2015). ¿Que son los precios hedónicos? Asociación de arquitectos tasadores de Chile A.G., 1, pp. 6.

Cerda, (2003). Valoración económica del medio ambiente. II Curso Instrumentos de Mercado y Fuentes de Financiamiento para el Desarrollo Sostenible. Cartagena de Indias, Colombia.

División de Estadística de las Naciones Unidas (UNSD) (2021). Tomar medidas urgentes para combatir el cambio climático y sus impactos, Departamento de Asuntos Económicos y Sociales (DESA). En: https://unstats.un.org/sdgs/report/2020/goal-13/

Figueroa, (2013). Crecimiento económico y medio ambiente. Revista CEPAL. 109, pp. 20-42.

Lever, (2000). Determinantes del precio de la vivienda en Santiago: Una estimación hedónica. Paper. Editorial Santiago, Chile

Mendieta, (1999). Manual de valoración económica de bienes no mercadeables: aplicaciones de las técnicas de valoración no mercadeables y el análisis costo beneficio y medio ambiente. Universidad de los Andes, Repositorio Institucional. Colombia.

Moreno y Alvarado. (2011). El entorno social y su impacto en el precio de la vivienda: un análisis de precios hedónicos en el área metropolitana de Monterrey. Trayectorias, 14(33), pp.131-147.

Organización de las Naciones Unidas (ONU). (2015). El Acuerdo de París, de Naciones Unidas acción por el clima. En: https://www.un.org/es/climatechange/paris-agreement

ONU (2019). La Agenda para el Desarrollo Sostenible, de Organización de las Naciones Unidas (ONU). En: https://www.un.org/sustainabledevelopment/es/development-agenda/

Rangel, ; Durán, ; Gómez, ; Ferro, ; Barranco, \& Sánchez, (2013) Valoración económico-ambiental de recursos naturales seleccionados en la cuenca del río Guanabo, La Habana, Cuba. REVIBEC. 20, pp. 45-55.

Secretaría de desarrollo sustentable (2021). Reporte del estado de la calidad del aire en el área metropolitana de Monterrey de Secretaría de desarrollo sustentable. En: https://www.nl.gob.mx/ 